\title{
Magnetic Null-Pairs within Magnetic Reconnection Ion Diffusion Region in the Magnetotail: A Case Study
}

\author{
Shiyou Li \\ School of Electronic Information, Hunan University of Information Technology, Changsha, China \\ Email: toneylab@163.com
}

How to cite this paper: Li, S.Y. (2019) Magnetic Null-Pairs within Magnetic Reconnection Ion Diffusion Region in the Magnetotail: A Case Study. International Journal of Geosciences, 10, 967-980. https://doi.org/10.4236/ijg.2019.1011055

Received: August 21, 2019

Accepted: October 28, 2019

Published: October 31, 2019

Copyright (อ 2019 by author(s) and Scientific Research Publishing Inc. This work is licensed under the Creative Commons Attribution International License (CC BY 4.0).

http://creativecommons.org/licenses/by/4.0/

\section{(c) (i) Open Access}

\begin{abstract}
The 3-dimentional structure of magnetic reconnection ion diffusion region has been studied in this paper. Steady magnetic null-pair structure is found among the Cluster tetrahedron within a thin current sheet when magnetic reconnection takes place in the near-Earth magnetotail. Two magnetic null points in the null-pair are well coupled, with an angle of about $3 \sim 7^{\circ}$ between the spin line of one and the fan surface of the other. The magnetic null-pair detected in the ion diffusion region, is quasi-stable in spatial structure but fast evolved in time, consistent with the fast reconnection scenario. The spatially steady magnetic null-pair within the diffusion region of the collision less fast magnetic reconnection presents an advanced understanding of the magnetic reconnection process.
\end{abstract}

\section{Keywords}

Magnetic Reconnection, Magnetic Null Point, Null-Pair

\section{Introduction}

Being regarded as the most important mechanism of the energy release and momentum transportation in the space and the laboratory plasma, magnetic reconnection [1] [2] is complicated in both magnetic structure and physical processes. With multi-scale processes of different physics in the vicinity of the magnetic reconnection X-line (2-D) or the magnetic null point (3-D) [3]-[7] in the reconnection process, the magnetic configuration remains ambiguous even though it has been studied for decades. Most studies on the reconnection, both theoretical and simulative, are in the regime of the ideal 2-D model. However, 
the reconnection taking place in the natural space is of 3-D structure, with magnetic null as the reconnection diffusion site. There are a lot of studies on the possible topology of reconnections in the $3-\mathrm{D}$ regime with or without magnetic nulls (e.g., [3]-[15]).

The magnetic null structure in the magnetic reconnection diffusion region (hereafter called "MRDR") has been studied in detail. Firstly, Xiao et al. [12] applied the null concept and arithmetic to space physics to interpret the 3-D magnetic reconnection in the near-earth central current sheet. They went even further to investigate the geometry of the magnetic null in detail and found a null-pair around which lower-hybrid wave was found [13]. Secondly, He et al. [14] developed a new method to construct the magnetic topology around a magnetic null in the magnetotail. The method is developed for reconstructing the local magnetic field based on the four-point measurement from Cluster tetrahedron. The method makes use of a fitting function with 10 fitting parameters in 10 spherical harmonic functions and another two in the Harris current sheet model, thus matching the 12 observed field components. Thirdly, He et al. [15] studied the electron dynamics in the region close to the null point, observing electron beams in two directions as seen in the pitch angle distributions (PAD) of electron differential energy flux measured by SC2/PEACE, and Deng et al. [16] have studied the structure of magnetic null and the micro process of wave turbulence that are very close to the coupled magnetic null in the diffusion region.

However, some issues remain ambiguous. For example, what is the difference between the structure of 2-D reconnection model (X-line) and 3-D geometry (magnetic null)? How does the 3-D reconnection develop? What other phenomena may arise and develop during the 3-D reconnection and what roles they play in the development of the 3-D reconnection? In present work, we apply the Poincare index [17] and the magnetic null fitting method [14] to the study the geometry of magnetic null-pairs, which are observed within the MRDR on 10 September 2001. This event has been studied by Wang et al. [18] [19] and Li et al. [20] extensively which mainly focused on the energetic electrons within the reconnection diffusion region [18] and electron pitch angle distributions in the vicinity of the $\mathrm{X}$ line and the outflow region [19]. We have also investigated the waves and particle dynamics around the magnetic null point in our previous work [20]. We will go further on this event to extend our understanding on the magnetic structure of the magnetic reconnection diffusion region.

Data used in this paper include Spin-resolution (4 seconds) data from the FluxGate Magnetometer (FGM) instrument [21] and the Cluster Ion Spectrometry (CIS) [22]. The full resolution $(22.46 \mathrm{~Hz})$ data is from the FGM. This paper is arranged as follows. In Section 2, the magnetic reconnection process is reviewed briefly. In Section 3, geometry of the magnetic null-pair in the diffusion region is studied in detail. Summary and discussion will be addressed in the final section. 


\section{Magnetic Reconnection Overview}

The magnetic reconnection event occurred on 10 September 2001, when Cluster is located in the near-earth tail at $(-19.29,2.19,0.46) \mathrm{R}_{\mathrm{E}}$ in the Geocentric Solar Magnetospheric (GSM) coordinate system (the same coordinate system will be used throughout this paper), has been studied extensively by Wang et al. [18] [19]. The crossing of all the spacecrafts to the ion diffusion region during the time interval of 07:50 - 08:05 UT has been confirmed by the key observation characteristics in Wang et al. [18] [19] which include 1) A reversal of high-speed flow $V_{x}$ (from negative to positive) coincides with a reversal of $B_{z}$ (from negative to positive); 2) Out-of-plane Hall magnetic field $B_{y}$ is observed by the four satellites of the Cluster.

In this study, we will focus on the structure of the ion diffusion region. We will brief review this event by plotting the $\mathrm{X}$ - and $\mathrm{Z}$-component of the magnetic field ( $B_{x}$ and $B_{z}$ ), X-component of plasma velocity $\left(V_{x}\right)$, plasma beta $(\beta)$, current density $\left(J_{x y z}\right)$ and the half thickness of the current sheet $\left(H_{C S / 2}\right)$ from 07:50 UT to 08:05 UT in the top part of Figure 1. Note that the plasma flow reversal takes place at around 07:57 UT, indicating that the satellites have crossed the ion diffusion region from tail ward side to earthward side in a rather short time interval. The four spacecrafts are directly crossing the ion diffusion region with $\mathrm{C} 1, \mathrm{C} 2$ and $\mathrm{C} 4$ mainly from the northern side of the current layer $\left(B_{x}>0\right.$, Figure 1(a)) and C3 from the southern side $\left(B_{x}<0\right.$, Figure 1(a)).

Figure $1(\mathrm{e})$ plots the current density $\left(J_{x y z}\right)$ within the Cluster tetrahedron which is calculated from the magnetic field vectors of the 4 terminals of the tetrahedron by the Cluster "Curlometer" analysis technique [23]. The Cluster "Curlometer"' analysis tool determines the current by calculating curl B from the magnetometer measurements on all four spacecraft [23]. The black, red and green lines represent the X-, Y-, and Z-components respectively. Though all the spacecrafts are not in the center of the current sheet $(20 \mathrm{nT}>|\mathrm{Bx}|>10 \mathrm{nT})$, the current density $\left(J_{x y z}\right)$ is indeed in the barycenter of the Cluster tetrahedron, which may be in the neutral current sheet since the satellites are on both the northern and southern sides of it. During the time interval when the spacecrafts cross the ion diffusion region, large cross-tail currents (ref. to Figure 1(e), $\left.J_{y}=10 \sim 15 \mathrm{nA} / \mathrm{m}^{2}\right)$ in the neutral current sheet can be differentiated.

The half thickness of the current sheet $\left(H_{C S / 2}\right.$, Figure $\left.1(\mathrm{f})\right)$ is estimated [24] by pressure balance condition [25] providing that the spacecraft is in the plasma sheet boundary layer (PSBL). Figure 1(f) plots the half thickness of the current sheet $\left(H_{C S / 2}\right)$. As is seen, the current sheet becomes rather thin at several time points, such as 07:56 UT, 07:57:10 UT, and 07:58 UT. Thus, a tendency of "thickening-thinning-thickening-thinning..." in the variation of the current sheet can be inferred from the variation of the current sheet's half thickness as shown in Figure 1(e), suggesting the flapping or other kinds of motion of the current sheet [26]. During the small time interval around 07:57 UT, the plasma flow reversal is observed by $\mathrm{C} 1, \mathrm{C} 3$ and C4 (data in C2 are not available) and 


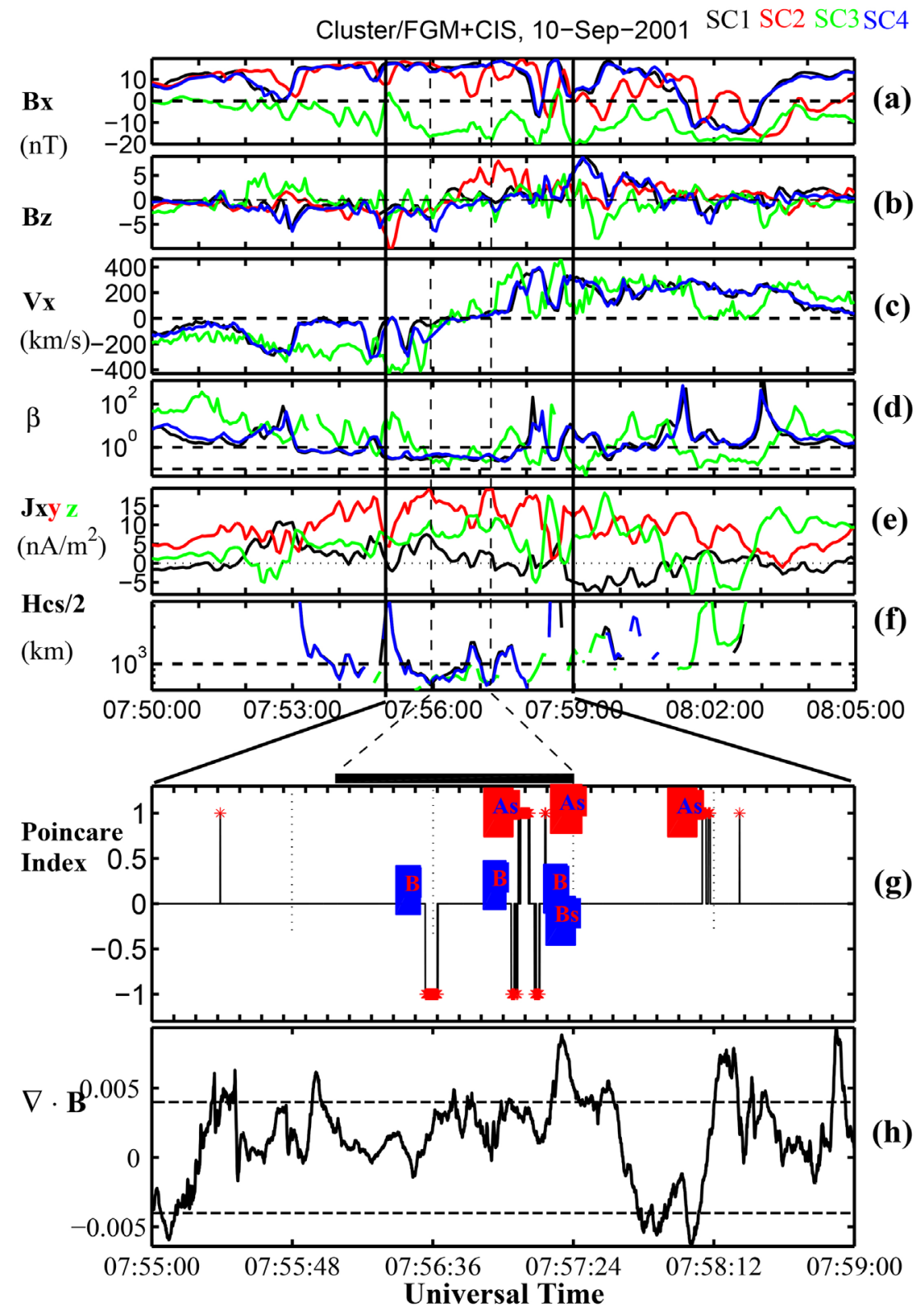

Figure 1. Overview of magnetic reconnection observation on 10 Sep. 2001. In each panel, black, red, green and blue lines represent the C1, C2, C3 and C4 observation. (a)-(b) X and Z-component of magnetic field; (c) X-components of the plasma velocity observed by CIS. The flow reversal occurs at 07:56 - 07:57 UT; (d) Plasma $\beta$. The two horizontal dotted lines represents $\beta=0.1$ and $\beta=1$, which indicate the plasma sheet boundary layer location of spacecraft; (e) The current density $\left(J_{x y z}\right)$ within the Cluster tetrahedron obtained by the "Curlometer" analysis technique; (f) Half thickness of the current sheet. The two vertical dashed lines indicate the time span (marked by the black rectangle in Figure 1(f)) for study; (g)-(h) Poincare index and corresponding $\nabla \cdot B$ in the time span from 07:56 UT to 07:59 UT.

meanwhile, the half thickness of the current sheet reduces severely to $<500 \mathrm{~km}$ (Figure 1(f)). Magnetic reconnection which takes place in a thin current sheet seems more likely to be three-dimensional [10] [27]. 
Forming a tetrahedron in space, the Cluster mission provides data from four similarly instrumented spacecraft and thus provides unique opportunities to study the 3-dimentional structures within the ion diffusion region of magnetic reconnection. The Poincar'e-index method, which was originally introduced by Greene [17] and developed by Zhao et al. [28], has been successfully employed to infer the presence of a true magnetic null point [12] [13] [14] [15]. In bottom part of Figure 1, the Poincare index and the corresponding $\nabla \cdot B$ from 07:55:00 UT to 07:59:00 UT by applying the Poincar'e-index method are shown. With poincare index of +1 or -1 , magnetic null points are included by the Cluster tetrahedron during this time (Figure $1(\mathrm{~g})$ ). The corresponding $\nabla \cdot B$ (Figure $1(\mathrm{~h})$ ) is very small (from -0.005 to 0.005 , the values indicated by the two horizontal dotted lines), confirming that the singular points are physical magnetic nulls. In this paper, we will mainly study the magnetic structure within reconnection diffusion region at around 07:57 UT.

\section{Geometry of Magnetic Null in the Diffusion Region}

\subsection{Magnetic Null-Pairs in the Reconnection Diffusion Region}

The magnetic structure around the reconnection diffusion region is much more complex than that in the ideal 2-dimensional model. In the case presented here, as indicated in Figure 1(f), the half thickness of the Current Sheet decreases rapidly to about $400-500 \mathrm{~km}$ when the flow reverses at about 07:57 UT. The thickness of the thin CS is as small as the scale of the ion inertial length $d_{i}\left(d_{i}-\right.$ $360 \mathrm{~km}$ ). Magnetic reconnection which takes place in a thin current sheet seems more likely to be three-dimensional [17] [28]. In this section, we will study the 3-D reconnection structure by employing the FGM data with a resolution of $22.46 \mathrm{~Hz}[21]$.

There are six types of null in space, i.e., the X-type and O-type in 2-D; A-type and B-type in 3-D and As-type and Bs-type in 3-D. The type of null is determined by the eigenvalues of the $\nabla \vec{B}$ matrix [5]. One can refer to Dorelli et al. [11] for details on the determination for these types of magnetic null structure. The magnetic nulls indicated by the Poincare index as shown in Figure $1(\mathrm{~g})$ are almost observed in the time span when plasma flow reverses (Figure 1(c)). They can be divided into 3 groups according to the observation time, namely N1 (07:56:33.3 - 07:56:37.8 UT), N2 (07:57:02.7 - 07:57:14.6 UT) and N3 (07:58:08.0 - 07:58:10.8 UT). For N1, the Poincare index for all the data points is -1 ; and it is +1 for N3. For N2, there are +1 and -1 with fast changes, which suggests complex structure in $\mathrm{N} 2$.

We classify the null types into A-, B-, As- and Bs-types according to the eigenvalues of the $\nabla \vec{B}$ matrix [5]. The field lines along the spine line of an A-type null direct out from the null, while field lines in the fan plane direct toward the null. And for the B-type null, the field line direction is opposite to the A-type null. The field lines around the As- and Bs-types are more complex than those in the A- and B-type nulls, with a spiral structure. Therefore, the type of 
magnetic null can be verified. Group N1 is of type-B, N3 is of type-As, and N2 contains types B-As-B (Bs)-As. The types of the null points are also marked in Figure $1(\mathrm{~g})$ and shown in Table 1. Note that in Table 1, " $\mathrm{j}$ " is the current density calculated by the Curlometer technique [23] and the "spine" and "fan" denote the $\gamma$-line and the $\Sigma$-surface of the magnetic null geometry, respectively.

The 3-dimentional magnetic reconnection occurs on a separator line that is analogous to a magnetic reconnection X-line in 2-dimensional scenario. The legs of this line are called separatrices in 2-dimensional. They correspond to fans ( $\Sigma$-surfaces) bounded by spines ( $\gamma$-line) that emerge from the nulls in 3 -dimentional [8]. For each magnetic null, the geometry of $\Sigma$-surface and $\gamma$-line and the current density are studied. The angles between the current density and the $\Sigma$-surface and $\gamma$-line are used to help us to understand the null geometry. The results are shown in Table 1, in which columns 3 to 6 are the type of null points, angle between $\mathrm{j}$ and $\Sigma$-surface, angle between $\mathrm{j}$ and $\gamma$-line, and the averaged value of the corresponding $\nabla \cdot B$.

It is very interesting that there are 4 null points (they are hereafter named as $\mathrm{N} 2 \mathrm{a}, \mathrm{N} 2 \mathrm{~b}, \mathrm{~N} 2 \mathrm{c}$ and N2d as in Table 1) in N2, separated by small time intervals. This suggests a very complex magnetic structure in the magnetic reconnection diffusion region. To study the relationship between 2 adjacent magnetic nulls, we take N2a-N2b, N2b-N2c, N2c-N2d as candidate magnetic null-pairs [13] [14] [15] [16]. For each candidate null-pair, we calculate the angle between the current density to the $\gamma$-line and the $\Sigma$-surface, and the angles between the $\gamma$-line and the $\Sigma$-surface. The results are summarized in Table 2 (N1 and N3 are analyzed with the same procedure, but we only want to illustrate the characteristics for these two magnetic nulls).

The angles are calculated under the assumption that there are two different magnetic nulls adjacent to each other, and the "1" and " 2 " denote the first and the second ones, respectively. " $\mathrm{j}$ " is the current density; "spine" and "fan" represent the $\gamma$-line and the $\Sigma$-surface for the corresponding magnetic null. Taking the N2a-N2b pair for an example, the angle between the $\gamma$-line of N2a (B-type null) and the $\Sigma$-surface of N2b (As-type null) is as small as $3.36^{\circ}$, indicating that the $\gamma$-line of the N2a null is almost in the $\Sigma$-surface of N2b; the angle between the As-type null $\gamma$-line and the $\Sigma$-surface of the B-type null is $34.72^{\circ}$. This angle is not very small. It is suggested to be caused by the deviation brought about from the spiral characteristic of the $\gamma$-line of the As-type null. Thus, this magnetic null-pair is confirmed to be a "B-As" coupled null-pair. Figure 2 illustrates the magnetic null-pair of N2a-N2b. The rough dashed lines represent the $\gamma$-line.

The same analysis is performed for the magnetic null-pair of N2c-N2d, but with the result slightly different from that of the N2c-N2d pair with regard to the angles. For the N2b-N2c null-pair, the situation is almost the same as for the $\mathrm{N} 2 \mathrm{c}-\mathrm{N} 2 \mathrm{~d}$ pair, with the angle of the $\gamma$-line of the Bs-type null $(\mathrm{N} 2 \mathrm{c})$ and the $\Sigma$-surface of the As-type null (N2b) as small as $3.09^{\circ}$. Though the angle between the $\gamma$-line of the B (Bs) type null and the $\Sigma$-surface of the As-type null is not 
Table 1. Magnetic null structure observed at 07:55:00 - 07:59:00 UT on 10 September 2001.

\begin{tabular}{cccccc}
\hline Ni & time & type & Angle $(j$, fan $)$ & Angle $(j$, spine $)$ & $\nabla \cdot B$ \\
\hline N1 & $07: 56: 33.3-07: 56: 37.8$ & B & 9.46 & 82.83 & 0.0015 \\
N2a & $07: 57: 02.7-07: 57: 05.0$ & B & 37.61 & 77.26 & 0.003 \\
N2b & $07: 57: 05.2-07: 57: 09.1$ & As & 82.05 & 46.61 & 0.003 \\
N2c & $07: 57: 10.7-07: 57: 12.5$ & B (Bs) & 29.81 & 79.88 & 0.0015 \\
N2d & $07: 57: 14.2-07: 57: 14.6$ & As & 59.99 & 39.14 & 0.0021 \\
N3 & $07: 58: 08.0-07: 58: 10.8$ & As & 27.18 & 43.46 & -0.0015 \\
\hline
\end{tabular}

Table 2. Magnetic null-pair structure at 07:55:00 - 07:59:00 UT on 10 September 2001.

\begin{tabular}{cccccccc}
\hline null-pair & j_spine1 & j_fan1 & j_spine2 & j_fan2 & spine1_fan2 & spine2_fan1 & coupled \\
\hline N1 - N1 & 82.83 & 9.46 & 82.83 & 9.46 & 34.36 & 34.36 & Single B \\
N2a - N2b & 77.26 & 37.61 & 46.61 & 82.05 & 3.37 & 34.72 & $\begin{array}{c}\text { B (Bs)-As } \\
\text { coupled }\end{array}$ \\
N2b - N2c & 46.61 & 82.05 & 79.88 & 29.81 & 24.07 & 3.09 & $\begin{array}{c}\text { As-(B) Bs } \\
\text { coupled }\end{array}$ \\
N2c- N2d & 79.88 & 29.81 & 39.14 & 59.99 & 7.41 & 24.37 & $\begin{array}{c}\text { (B) Bs-As } \\
\text { coupled }\end{array}$ \\
N3 - N3 & 43.46 & 27.18 & 43.46 & 27.18 & 31.47 & 31.47 & Single As \\
\hline
\end{tabular}

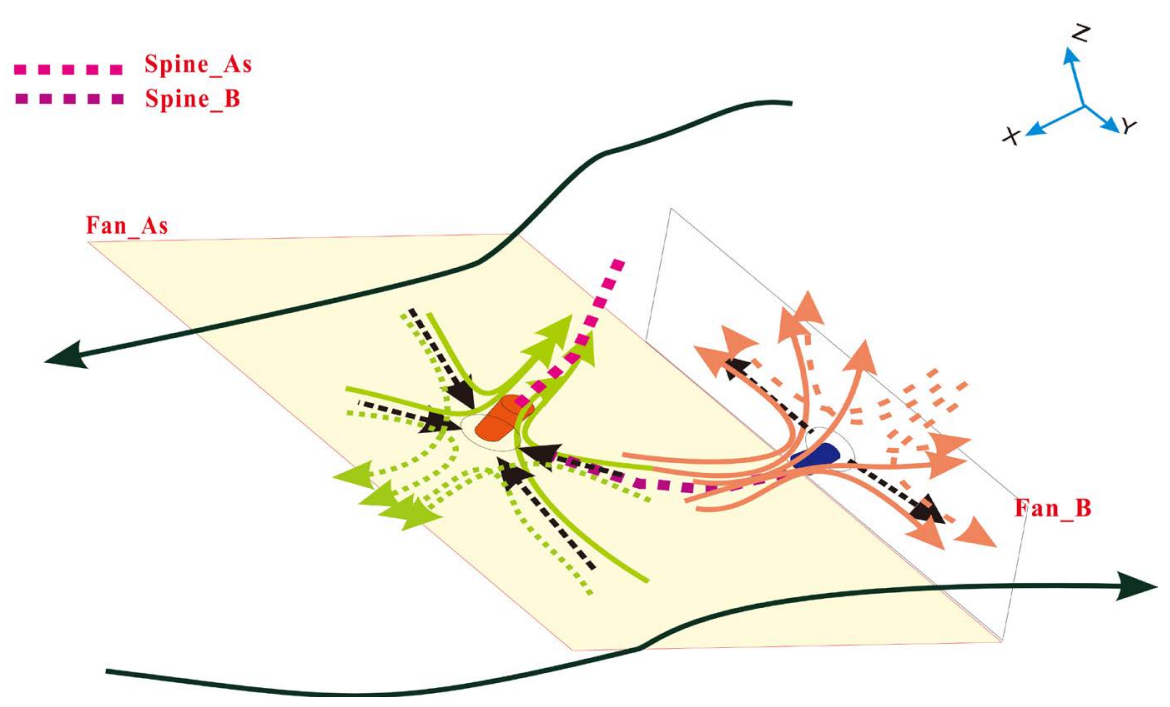

Figure 2. Cartoon for magnetic null-pair inclusion by the Cluster tetrahedron at 07:57:04 UT. Cluster includes the B-type null of the magnetic null-pair. The oblique orange cylinder represents an As-type magnetic null, while the blue one represents a B-type null. The parallelograms are the Fan-surfaces ( $\Sigma$-surface) of the corresponding magnetic null, while the thick dashed lines as shown in the left-upper inlet represent the spine line of the magnetic null. The spine line of B-type null is almost located in the $\Sigma$-surface of As-type null. The thick black solid lines in the outermost indicate the direction of the magnetic field lines in the northern PSBL and southern PSBL. 
exactly the same as the former null-pair addressed above, their analogy can still be reliable. The relationship between the two groups of the magnetic null-pairs will be dressed in the following.

\subsection{Magnetic Fitting of Magnetic Null-Pairs}

The magnetic null observation during the time span from 07:56:33.3 UT to 07:58:10.8 UT was divided into 3 stages: Stage-1: 07:56:33.3 - 07:56:33.8 UT (N1); Stage-2: 07:57:05.2 to 07:57.14.6 UT (N2); Stage-3: 07:58:08.0 to 07:58:10.8 UT (N3). It was found that in stage-1 (N1), it is a single B-type magnetic null; in stage-3 (N3), it is a single As-type magnetic null. However, in Stage-2, there are 3 magnetic null-pairs in which the angle between the $\gamma$-line of one null and the $\Sigma$-surface of the other is rather small. The 3 magnetic null-pairs are: 1) B (Bs)-As coupled magnetic null-pair; 2) As-(B) Bs coupled magnetic null-pair; 3) (B) Bs-As coupled magnetic null-pair. The difference between topologies of these 3 magnetic null-pairs is very small. This small difference suggests that the magnetic geometry can change itself slightly during the reconnection process at this time. In this subsection, further study of the magnetic null geometry will be done.

To study the relationship among these 3 above-mentioned magnetic null-pairs, the magnetic field fitting method [14] is applied here to reconstruct the field line around the magnetic null. The fitting method is restated below.

The magnetic fitting method was designed by $\mathrm{He}$ et al. [14] on the year of 2008. This method is to fit the recorded 4 vectors with 12 magnetic field components simultaneously measured by the Cluster satellites. There are 12 functions in the fitting model, including ten spherical harmonic functions and a function taken from the Harris current sheet model by Harris [29] in 1962, together with a constant background field. They adopted the spherical harmonic functions as part of the fitting model for their convenience of describing a potential field. Considering the special feature of magnetic field configuration in the magnetotail, we add the Harris current sheet function with a constant background field to the fitting function. Such a fitting can be expressed as

$$
\left(\begin{array}{c}
B_{r} \\
B_{\theta} \\
B_{\phi}
\end{array}\right)=\left(\begin{array}{c}
\widetilde{B_{R}} \\
\widetilde{B_{\theta}} \\
\widetilde{B_{\phi}}
\end{array}\right)+T_{x y z \rightarrow r \theta \phi} \cdot\left(\begin{array}{c}
B_{0} \tanh \left(\frac{z-z_{0}}{L_{z}}\right)+B_{1} \\
0 \\
0
\end{array}\right)
$$

where $\left(B_{r}, B_{\theta}, B_{\phi}\right)$ represent 3 magnetic field components at a spatial position $(r, \theta, \phi)$ in a spherical coordinate system with its origin at the center of the Earth. The first term on the right-hand side (RHS) of Equation (1), $\left(\widetilde{B_{r}}, \widetilde{B_{\theta}}, \widetilde{B_{\phi}}\right)$, is the contribution from the spherical harmonic series describing a potential field, as shown below in Equation (2). The transform matrix $T_{x y z \rightarrow r \theta \phi}$ converts a vector field from the GSM to a geocentric spherical coordinate system. The magnetic field in the Harris current model plus a constant background field is in the $\mathrm{x}$-direction as shown in Equation (1). Expression for $\left(\widetilde{B_{r}}, \widetilde{B_{\theta}}, \widetilde{B_{\phi}}\right)$ reads 


$$
\left(\begin{array}{l}
\widetilde{B_{R}} \\
\widetilde{B_{\theta}} \\
\widetilde{B_{\phi}}
\end{array}\right)=\left(\begin{array}{l}
\sum_{n} \sum_{m}[-(n+1)]\left(\frac{R_{e}}{r}\right)^{n+2} \cdot\left(q_{n}^{m} \cos (m \varphi)+h_{n}^{m} \sin (m \varphi)\right) \cdot p_{n}^{m}(\cos \theta) \\
\frac{R_{e}}{r} \sum_{n} \sum_{m}\left(\frac{R_{e}}{r}\right)^{n+1} \cdot\left(q_{n}^{m} \cos (m \varphi)+h_{n}^{m} \sin (m \varphi)\right) \cdot(-\sin \theta) \cdot \frac{\partial}{\partial \theta}\left(p_{n}^{m}(\cos \theta)\right) \\
\frac{R_{e}}{r \sin \theta} \sum_{n} \sum_{m}\left(\frac{R_{e}}{r}\right)^{n+1} \cdot\left(q_{n}^{m}(-m) \cdot \sin (m \varphi)+h_{n}^{m} \cdot m \cdot \cos (m \varphi)\right) \cdot p_{n}^{m}(\cos \theta)
\end{array}\right)
$$

where $q_{n}^{m}$ and $h_{n}^{m}$ are the coefficients in the spherical harmonic series, and $p_{n}^{m}$ is the associated Legendre function of degree $\mathrm{n}$ and order $\mathrm{m}$, with $[\mathrm{n}, \mathrm{m}]=$ $\{[1,1],[2,1],[\{2,2\}],[3,1],[3,2]\}$. The second term on the RHS of Equation (1) is designed specifically to represent the magnetotail environment.

Figure 3 shows the fitting result of this structure when the magnetic null is included by the tetrahedron at 6-time points, corresponding to N1 (a), N2 (b d), N3 (e and f) stages. The fitting parameters are: half thickness of current sheet is $500 \mathrm{~km}$ (refer to Figure 1(e)); the normal direction of the current sheet is $(0$, 0,1 ). Figure 3(a) shows a single magnetic null included by the Cluster tetrahedron, which well matches the observation of N1. Figures 3(b)-(d) show the fitting structure corresponding to the 3 sub-stages in N2 stage. Fitting result in this stage shows that magnetic null-pair does exist within the tetrahedron of Cluster. Figure 3(e) and Figure 3(f) show the fitting results corresponding to the N3 stage. The magnetic null-pair still exists in Figure 3(f), with only a part of it included by the Cluster tetrahedron. For those single magnetic nulls shown in Figure 3, as the magnetic null-pair is steady observed in stage 2, each of them can be suggested to be one part of a magnetic null-pair.

\subsection{Temporal Evolution of Magnetic Null-Pairs}

As is addressed above, some magnetic nulls form within the diffusion region crossing during time span from 07:56:33.3 UT to 07:57:14.6 UT. We have divided the null observation into 3 stages in the observation time span from 07:56:33.3 UT to 07:58:10.8 UT (stage 1: 07:56:33.3 - 07:56:33.8 UT (N1); stage 2: 07:57:05.2 to 07:57.14.6 UT (N2); stage 3: 07:58:08.0 to 07:58:10.8 UT (N3)).

Provided that the 3-D magnetic reconnection takes place in the space at a fixed time point, forming a magnetic null-pair as shown in Figure 2. In the first stage, Cluster moves into the diffusion region, and includes one part of the null-pair, which is presented to be the B-type null during 07:56:33.3 - 07:56:33.8 UT as seen in Figure 3(a). During this time span, the magnetic null structure is stable, only with its B-part included in the cluster tetrahedron. After that, the magnetic reconnection pauses temperately. About 20 seconds later, the magnetic reconnection re-occurs, forming the magnetic null-pair again. Firstly, one part of the null-pair (B-type null) is included by the Cluster tetrahedron, ref. to the illustrating drawing in Figure 2; and as the spacecrafts moving forward, the other part of the null-pair (A or As-type) is in turn included (Figure 3(c)). The magnetic null-pair lasts only several seconds. It moves from left to right (in 


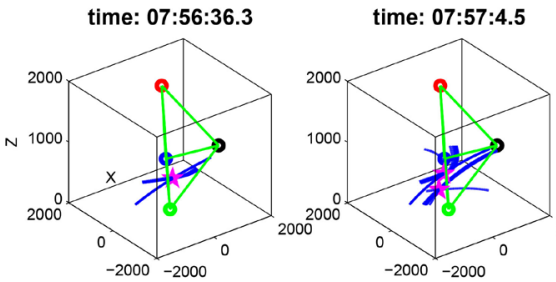

(a) $\uparrow$

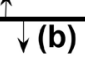

time: $07: 56: 37.0$
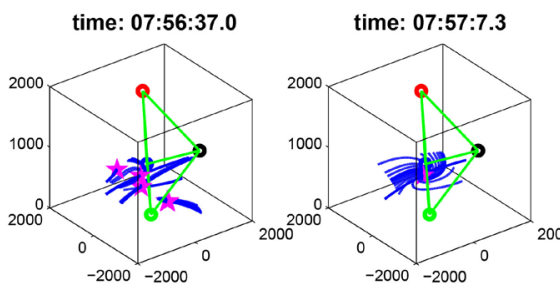

(c) ${ }^{\wedge}$
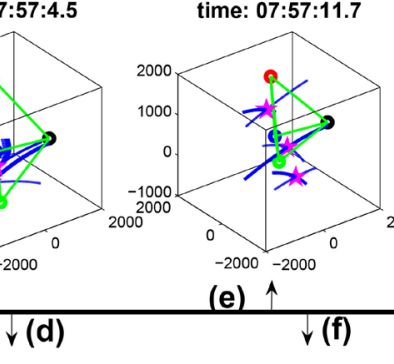

(e) $\uparrow$

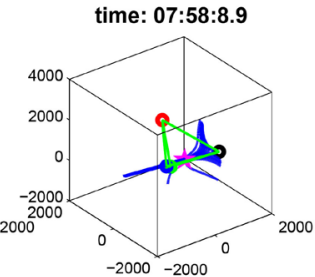

(a)

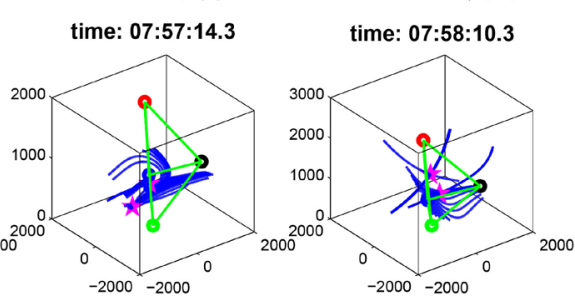

Figure 3. Magnetic field structure fitting in different stages of the observation, corresponding to the N1 (a), N2 (b - d), N3 (e) and (f) stages. The fitting parameters are: half thickness of current sheet is $500 \mathrm{~km}$; the normal direction of the current sheet is set as $(0$, $0,1)$.

Figure 3) and again, after ceasing for about 5 seconds, the reconnection re-occurs and form a new magnetic null-pair, which is detected by the tetrahedron during 07:57:10.7 - 07:57:14.6 UT (Figure 3(d)). In the third stage from 07:58:08.0 UT to 07:58:10.8 UT (Figure 3(e), Figure 3(f)), the results in Table 2 tell the same story for the As-type part of the magnetic null-pair of the magnetic reconnection diffusion region as what is addressed in the first stage.

The analysis above suggests that the magnetic null-pair, which is detected in the magnetic reconnection diffusion region, is a quasi-stable in spatial structure but with fast evolution in time. The temporal evolution and spatial quasi-stability of the magnetic null-pair shed a new light on the magnetic diffusion geometry in the reconnection region.

\section{Conclusion and Discussion}

We have studied the magnetic diffusion structure in regimes of 2-D ideal reconnection model and 3-D magnetic null as the reconnection taking place in the neutral current sheet in the near-earth tail on 10 Sep. 2001. Main points are summarized as follows:

1) Many magnetic null points have been detected by the Cluster in the diffusion crossing time. The $\mathrm{N} 1$ from 07:56:33.3 - 07:56:33.8 UT is a B-type single magnetic null; the N2 from 07:57:05.2 UT to 57.14.6 UT is composed of 4 magnetic nulls, which are regarded to be the coupled magnetic null-pair according to the angle between the $\gamma$-line of one and the $\Sigma$-surface of the other for each null-pair. N3 is also a single magnetic null (As type).

2) The magnetic field fitting result around the magnetic null or null-pair in the reconnection diffusion region has shown good magnetic structure around 
the null in different stages. The small difference in magnetic null-pairs suggests that the magnetic spatial topology in the reconnection diffusion region is very steady. For spatial geometry, the magnetic field forms a steady magnetic null-pair (B-As) in the magnetic diffusion region, suggesting a steady spatial geometry for the 3-D reconnection.

The 3-dimensional geometry of the magnetic null-pair, in this case, is different with the case on 15 September 2001 studied by Xiao et al. [12]. There is only one single magnetic null in the case. Though it can be inferred to be one part of the magnetic null-pair from the magnetic lines as in our case (N1), the two are not the same because of the lack of other parts of the magnetic null-pair. A magnetic null-pair is more stable than a single magnetic null in a 3-D magnetic regime. Our case is also different from the A-B-As coupled magnetic null structure in 1 October 2001 introduced by Xiao et al. [13] and studied in detail by $\mathrm{He}$ et al. [15] and Deng et al. [16]. The magnetic null-pair in the presented case evolves with time and is detected by Cluster time again, and therefore reinforces the topology modification during the relative stable 3-D magnetic geometry.

In Figure 1(g), when $\mathrm{C} 1 \mathrm{C} 3$ and $\mathrm{C} 4$ are located in the outflow region (Figure 1 (c), $|\mathrm{Vx}| \sim 300-400 \mathrm{~km} / \mathrm{s})$, there are several places where Poincare indexes were 1. They denote that there were also magnetic nulls there. However, this is just one single magnetic null, or at least it is one part of the magnetic null-pairs, which is included by the Cluster tetrahedron. Though the satellites are mainly located in the outflow region, however, the $\mathrm{C} 3$ maybe go back to the other side since $\mathrm{Vx}$ (c3) decreases rapidly to zero at this time. Thus it is possible for the magnetic nulls to be included by the tetrahedron.

The current sheet during this time is unstable. As addressed in part 2, the current sheet presents a kink-like configuration with a tendency of "thickening, thinning, thickening, and thinning" of the current sheet variation. The thickness of the current sheet is less than $1000 \mathrm{~km}$ in the magnetic null observation, i.e., the magnetic null-pair forms a steady 3-D reconnection structure in a thin current sheet. Moreover, SC2 is located northern relative to the other 3 satellites. Thus the relative location of SC2 to SC3 is further than those of SC1 and SC4 to SC3. However, the X-component of magnetic field $\left(B_{x}\right)$ detected by SC2 is smaller than what detected by the SC1 and SC4 during the time interval from 07:56:20 UT to 07:57:40 UT. This suggests a tilt of the current sheet X-Y plane. Thus, the current sheet during this time is completely in the 3-D regime, which might be a response or an exciter of the 3-D magnetic structure in the 3-D magnetic reconnection process. The large scale of the current sheet associated with the 3-D geometry of magnetic diffusion region remains to future work.

\section{Acknowledgements}

The authors thank all the members of the Cluster team for the high quality data and successful operation. This work is supported by the Scientific Research Projects of Hunan Education Department (No.18B572). 


\section{Conflicts of Interest}

The author declares no conflicts of interest regarding the publication of this paper.

\section{References}

[1] Parker, E.N. (1957) Sweet's Mechanism for Merging Magnetic Fields in Conducting Fluids. Journal of Geophysical Research, 62, 509-520. https://doi.org/10.1029/JZ062i004p00509

[2] Dungey, J.W. (1961) Interplanetary Field and the Auroral Zones. Physical Review Letters, 6, 47-48. https://doi.org/10.1103/PhysRevLett.6.47

[3] Büchner, J. and Kuska, J.P. (1996) Three-Dimensional Collisionless Reconnection through Thin Current Sheets: Theory and Self-Consistent Simulations. Proceedings of the 3 rd International Conference on Substorms, ESA SP-389, October 1996, 373.

[4] Büchner, J. and Kuska, J.P. (1997) Numerical Simulation of Three-Dimensional Reconnection Due to the Instability of Collisionless Current Sheets. Advances in Space Research, 19, 1817-1822. https://doi.org/10.1016/S0273-1177(97)00082-3

[5] Priest, E.R. and Titov, V.S. (1996) Magnetic Reconnection at Three-Dimensional Null Points. Philosophical Transactions of the Royal Society A: Mathematical, Physical and Engineering Sciences, 354, 2951-2992. https://doi.org/10.1098/rsta.1996.0136

[6] Schumacher, J., Kliem, B. and Seehafer, N. (2000) Three-Dimensional Spontaneous Magnetic Reconnection in Neutral Current Sheets. Physics of Plasmas, 7, 108-121. https://doi.org/10.1063/1.873786

[7] Stenzel, R.L., Griskey, M.C., Urrutia, J.M. and Strohmaier, K.D. (2003) Three-Dimensional Electron Magnetohydrodynamic Reconnection: I. Fields, Currents, and Flows. Physics of Plasmas, 10, 2780. https://doi.org/10.1063/1.1578998

[8] Lau, Y.T. and Finn, J.M. (1990) Three-Dimensional Kinematic Reconnection in the Presence of Teld Nulls and Closed Teld Lines. The Astrophysical Journal, 350 , 672-691. https://doi.org/10.1086/168419

[9] Parnell, C.E., Neukirch, T., Smith, J.M. and Priest, E.R. (1996) The Structure of Three-Dimensional Magnetic Neutral Points. Physics of Plasmas, 84, 245-271. https://doi.org/10.1063/1.871810

[10] Zhu, Z. and Winglee, R.M. (1996) Tearing Instability, Flux Ropes, and the Kinetic Current Sheet Kink Instability in the Earth's Magnetotail: A Three-Dimensional Perspective from Particle Simulations. Journal of Geophysical Research, 101, 4885-4898. https://doi.org/10.1029/95JA03144

[11] Dorelli, J.C., Bhattacharjee, A. and Raeder, J. (2007) Separator Reconnection at Earth's Dayside Magnetopause under Generic Northward Interplanetary Magnetic Field Conditions. Journal of Geophysical Research Space Physics, 112, A02202. https://doi.org/10.1029/2006JA011877

[12] Xiao, C.J., Wang, X.G., Pu, Z.Y., Zhao, H., Wang, J.X., Ma, Z.W., Fu, S.Y., Kivelson, M.G., Liu, Z.X., Zong, Q.G., Glassmeier, K.H., Balogh, A., Korth, A., Reme, H. and Escoubet, C.P. (2006) In Situ Evidence for the Structure of the Magnetic Null in a 3d Reconnection Event in the Earth's Magnetotail. Nature Physics, 2, 478-483. https://doi.org/10.1038/nphys342

[13] Xiao, C.J., Wang, X.G., Pu, Z.Y., Ma, Z.W., Zhao, H., Zhou, G.P., Wang, J.X., Kivelson, M.G., Fu, S.Y., Liu, Z.X., Zong, Q.G., Dunlop, M.W., Glassmeier, K.-H., Lucek, E., Reme, H., Dandouras, I. and Escoubet, C.P. (2007) Satellite Observations of 
Separator-Line Geometry of Three-Dimensional Magnetic Reconnection. Nature Physics, 3, 609-613. https://doi.org/10.1038/nphys650

[14] He, J.S., Tu, C.Y., Tian, H., Xiao, C.J., Wang, X.G., Pu, Z.Y., et al. (2008) A Magnetic Null Geometry Reconstructed from Cluster Spacecraft Observations. Journal of Geophysical Research, 113, A05205. https://doi.org/10.1029/2007JA012609

[15] He, J.S., Zong, Q.G., Deng, X.H., Tu, C.Y., Xiao, C.J., Wang, X.G., Ma, Z.W., Pu, Z.Y., et al. (2008) Electron Trapping around a Magnetic Null. Geo Physical Review Letters, 35, L14104. https://doi.org/10.1029/2008GL034085

[16] Deng, X.H., Zhou, M., Li, S.Y., Baumjohann, W., André, M., Cornilleauwehrlin, N., et al. (2009) Dynamics and Waves near Multiple Magnetic Null Points in Reconnection Diffusion Region. Journal of Geophysical Research Space Physics, 114, A07216. https://doi.org/10.1029/2008JA013197

[17] Greene, J.M. (1992) Locating Three-Dimensional Roots by a Bisect Ion Method. Journal of Computational Physics, 98, 194-198. https://doi.org/10.1016/0021-9991(92)90137-N

[18] Wang, R.-S., Lu, Q.-M., Guo, J. and Wang, S. (2008) Spatial Distribution of Energetic Electrons during Magnetic Reconnection. Chinese Physics Letters, 25, 3083-3085. https://doi.org/10.1088/0256-307X/25/8/093

[19] Wang, R., Lu, Q., Huang, C. and Wang, S. (2010) Multispacecraft Observation of Electron Pitch Angle Distributions in Magnetotail Reconnection. Journal of Geophysical Research Space Physics, 115, A01209. https://doi.org/10.1029/2009JA014553

[20] Li, S.Y., Zhang, S.F., Cai, H. and Yang, H.B. (2014) Concentration of Electrostatic Solitary Waves around Magnetic Nulls within Magnetic Reconnection Diffusion Region: Single-Event-Based Statistics. Earth, Planets and Space, 66, 161. https://doi.org/10.1186/s40623-014-0161-3

[21] Balogh, A., Dunlop, M.W., Cowley, S.W.H., Southwood, D.J., Thomlinson, J.G., Glassmeier, K.H., et al. (1997) The Cluster Magnetic Field Investigation. Space Science Reviews, 79, 65-91. https://doi.org/10.1023/A:1004970907748

[22] Reme, H., Cottin, F., Cros, A., et al. (1997) The Cluster Ion Spectrometry (CIS) Experiment. Space Sciences Review, 79, 303-350.

[23] Dunlop, M.W., Balogh, A., Glassmeier, K.H. and Robert, P. (2002) Four-Point Cluster Application of Magnetic Field Analysis Tools: The Curlometer. Journal of Geophysical Research, 107, 1384. https://doi.org/10.1029/2001JA005088

[24] Asano, Y., Mukai, T., Hoshino, M., Saito, Y., Hayakawa, H. and Nagai, T. (2003) Evolution of the Thin Current Sheet in a Substorm Observed by Geotail. Journal of Geophysical Research: Space Physics, 108, 1189.

https://doi.org/10.1029/2002JA009785

[25] Matsumoto, Y., Mukai, T., Saito, Y., Kokubun, S. and Hoshino, M. (2001) On the Pressure Balance in the Distant Magnetotail. Journal of Geophysical Research Atmospheres, 106, 25905-25918. https://doi.org/10.1029/2001JA900064

[26] Sergeev, V., Runov, A., Baumjohann, W., et al. (2003) Current Sheet Flapping Motion and Structure Observed by Cluster. GeoPhysical Review Letters, 30, 1327. https://doi.org/10.1029/2002GL016500

[27] Pritchett, P.L., Coroniti, F.V. and Decyk, V.K. (1996) Three-Dimensional Stability of Thin Quasi-Neutral Current Sheets. Journal of Geophysical Research, 101, 27413-27430. https://doi.org/10.1029/96JA02665

[28] Zhao, H., Wang, J.X., Zhang, J. and Xiao, C.J. (2005) A New Method of Identifying 
3d Null Points in Solar Vector Magnetic Fields. Chinese Journal of Astronomy and Astrophysics, 5, 443-447. https://doi.org/10.1088/1009-9271/5/5/001

[29] Harris, E.G. (1962) On a Plasma Sheet Separating Regions of Oppositely Directed Magnetic Field. Il Nuovo Cimento, 23, 115-121.

https://doi.org/10.1007/BF02733547 